\title{
A RIGIDITY THEOREM FOR QUATERNIONIC-KÄHLER MANIFOLDS
}

\author{
CLAUDE LEBRUN
}

(Communicated by Irwin Kra)

\begin{abstract}
Let $(M, g)$ be a compact quaternionic-Kähler manifold of dimension $\geq 8$ and positive scalar curvature. It is shown that $(M, g)$ has no nontrivial deformations through quaternionic Kähler manifolds.
\end{abstract}

Let $\left(M^{4 k}, g\right), k \geq 2$, be a Riemannian manifold whose holonomy group is a subgroup of $\operatorname{Sp}(k) \operatorname{Sp}(1)$; we say that $(M, g)$ is a quaternionic-Kähler manifold. Suppose moreover that $M$ is compact and that $g$ has scalar curvature $R>0$. The only known examples are symmetric spaces, and hence quite rigid. One may generalize this fact in the following direction: any deformation of $g$ through quaternionicKähler metrics is obtained by pulling back $g$ via a family of diffeomorphisms of $M$. We will prove this result by twistor methods.

To begin, let us recall Salamon's theory of quaternionic twistor spaces [S]. Each quaternionic-Kähler manifold $(M, g)$ has associated to it a complex manifold $Z$ together with a fibering $p: Z \rightarrow M$ whose fibers are Riemann spheres. Provided that $R>0, Z$ comes equipped with a Kähler-Einstein metric $h$ whose restriction to the orthogonal bundle $D$ of the vertical tangent space $\operatorname{ker} p_{*}$ is just the pull-back $p^{*} g$ of $g$. Moreover, the distribution $D$ is actually a complex contact structure: there exist local nonzero holomorphic 1 -forms $\theta$ whose kernel is precisely $D$ and such that $\theta \wedge(d \theta)^{\wedge k}$ is nowhere zero. If $L \rightarrow Z$ is the holomorphic line bundle $T Z / D$, we thus have an isomorphism $\kappa^{*} \cong L^{\otimes(k+1)}$ where $\kappa \rightarrow Z$ is the canonical line bundle.

Now since $h$ is Kähler-Einstein with positive scalar curvature, $\kappa^{*}$ and $L$ are positive line bundles, so that $\kappa^{*} \otimes L$ is also positive and $H^{1}(Z, \mathscr{O}(L))=0$ by the Kodaira vanishing theorem; the rigidity theorem will eventually follow from this observation. Notice that the positivity of $\kappa^{*}$ also implies that $Z$ is a smooth projective algebraic variety of Kodaira dimension $-\infty$, facts which we will not use here but which will probably be useful at some future date in classifying such quaternionic $(M, g)$.

We now prove the key technical lemma of this note.

PROPOSITION. Let $X$ be a compact complex contact manifold with contact distribution $D \subset T X ;$ let $L=T X / D$ be the contact line bundle of $X$, and suppose that $H^{1}(X, \mathscr{O}(L))=0$. Then any small complex contact deformation of $X$ is trivial, in the following sense: if $\pi: \hat{X} \rightarrow \mathbf{R}$ is a smooth proper map whose fibers $X_{t}=\pi^{-1}(t)$ are complex contact manifolds with complex contact structure depending smoothly

Received by the editors March 23, 1987 and, in revised form, June 15, 1987.

1980 Mathematics Subject Classification (1985 Revision). Primary 53C25; Secondary 32L20, $32 \mathrm{~L} 25$. 
on $t$, and if $X=X_{0}$, there is a neighborhood $I$ of $0 \in \mathbf{R}$ such that $\pi^{-1}(I) \cong X \times I$ in a fiber-wise complex contact manner. Moreover, if $H^{1}\left(X_{t}, \mathscr{O}\left(L_{t}\right)\right)=0$ for all $t \in \mathbf{R}$, where $L_{t} \rightarrow X_{t}$ is the contact line bundle analogous to $L \rightarrow X$, then $\hat{X} \cong X \times \mathbf{R}$ in a fiber-wise complex contact manner.

PrOOF. By the holomorphic version of Darboux's theorem $[\mathbf{A}], X$ has a holomorphic atlas of charts $\left\{\phi_{J}: U_{J} \rightarrow \mathbf{C}^{2 k+1}\right\}$ via which the contact distribution becomes the orthogonal space of

$$
\theta=d z^{2 k+1}+\sum_{m=1}^{k} d z^{m} \wedge d z^{m+k} .
$$

We will now think of the canonical projection $T X \rightarrow T X / D$ as a twisted 1-form $\theta_{X} \in \Gamma\left(X, \Omega^{1}(L)\right)$ which becomes $\phi_{J}^{*} \theta$ in a local trivialization of $L$ over the open set $U_{J} \subset X$.

Let $\mathscr{C}$ be the sheaf of holomorphic vector fields $V$ on $X$ having the property that $L_{V} \mathscr{O}(D) \subset \mathscr{O}(D)$; elements of $\mathscr{C}$ are called infinitesimal complex contact transformations. There is a natural isomorphism between $\mathscr{C}$ and $\mathscr{O}(L)$ given by $V \rightarrow V\lrcorner \theta_{X}$, whose inverse is obtained in local coordinates on $U_{j}$ by solving the equations

$$
V\lrcorner \theta=f, \quad V\lrcorner d \theta \equiv-d f \quad \bmod \theta,
$$

where $f \theta$ is any given local section of $L$; we shall denote this inverse by $\mu: \mathscr{O}(L) \rightarrow$ $\mathscr{C} \subset \mathscr{O}(T X)$.

Now equip $\hat{X}$ with charts $\hat{\phi}_{J}: \hat{U}_{J} \rightarrow \mathbf{C}^{2 k+1} \times \mathbf{R}$ which take open sets in $X_{t}$ to open sets in $\mathbf{C}^{2 k+1} \times\{t\}$ in such a way as to take $D_{t}$ to the orthogonal space of $\theta$. We may then consider the transition functions $\phi_{J K}: \phi_{K}\left(U_{J} \cap U_{K}\right) \rightarrow \phi_{J}\left(U_{J} \cap U_{K}\right)$ given by $\phi_{J K}=\phi_{J} \circ \phi_{K}^{-1}$ and let $V_{J K}(t) \in \Gamma\left(\pi^{-1}(t) \cap U_{J} \cap U_{K}, \mathscr{O}\left(\mathscr{C}_{t}\right)\right)$ be defined by

$$
V_{J K}(t)=\left(\phi_{J}^{-1}(t)\right)_{*} \frac{d}{d t} \phi_{J K}(t)
$$

where $\phi_{J K}(t)$ maps one subregion of $\mathbf{C}^{2 k+1}$ biholomorphically to another by $\phi_{J K}(t)(z)=\phi_{J K}(z, t), t \in \mathbf{R}$. Since $V_{J K}$ is an infinitesimal complex contact transformation on $U_{J} \cap U_{K}$, we may define $\theta_{J K} \in \Gamma\left(U_{J} \cap U_{K}, \mathscr{O}\left(L_{t}\right)\right)$ by

$$
\theta_{J K}(t)=\mu_{t}^{-1}\left(V_{J K}(t)\right) \text {. }
$$

To prove the proposition, we now proceed as in Kodaira-Spencer theory $[\mathbf{K}]$ and extract a finite subcover of $X_{t}$ from $\left\{U_{J} \cap \pi^{-1}(t)\right\}$, viewing $\theta_{J K}(t)$ as a Čech cocycle for $H^{1}\left(X_{t}, \mathscr{O}\left(L_{t}\right)\right)$. For $t$ in some neighborhood $I$ of 0 , the hypothesis $H^{1}(X, \mathscr{O}(L))=0$ implies that $H^{1}\left(X_{t}, \mathscr{O}\left(L_{t}\right)\right)=0$ by the upper semicontinuity of dimension, and we can choose sections $\theta_{J} \in \Gamma\left(U_{J}, \mathscr{O}\left(L_{t}\right)\right)$ with $\theta_{J K}(t)=\theta_{J}(t)-$ $\theta_{K}(t)$. We thus have vector fields $V_{J}(t)=\mu_{t}\left(\theta_{J}(t)\right)$ in $\mathscr{C}_{t}$ such that $V_{J K}=V_{J}-V_{K}$.

On $\phi_{J}\left(U_{J} \cap U_{K}\right)$, consider the ordinary differential equation

$$
d \hat{Z} / d t=-\phi_{J *} V_{J}, \quad \hat{Z}(Z, 0)=Z,
$$

where $\hat{Z}(Z, t) \in \mathbf{C}^{2 k+1}$ for $Z \in \mathbf{C}^{2 k+1}, t \in \mathbf{R}$, whose solutions define a biholomorphism from a neighborhood of $\phi_{J}\left(\pi^{-1}(t) \cap U_{J}\right)$ in $\phi_{J}\left(U_{J}\right)$ to another neighborhood of $\phi_{J}\left(\pi^{-1}(t) \cap U_{J}\right)$. Replacing $(Z, t)$ with the new coordinates $(\hat{Z}, t)$ be refining the cover, one obtains new transition functions which are independent of $t$. The 
proposition follows by noticing that this gives rise to isomorphisms of $\pi^{-1}\left(I_{\alpha}\right)$ with $X_{t \alpha} \times I_{\alpha}$ for intervals $I_{\alpha}$ covering $I$.

Notice that if $H^{1}\left(X_{t}, \mathscr{O}\left(L_{t}\right)\right)=0$ for all $t \in \mathbf{R}$, we may take $I=\mathbf{R}$. Q.E.D.

With this tool in hand, we may prove our rigidity theorem:

THEOREM. Let $M$ be a compact $4 k$-manifold, $k \geq 2$, and let $\left\{g_{t}\right\}$ be a family of quaternionic-Kähler metrics on $M$ of fixed volume depending smoothly on $t \in \mathbf{R}$. If $g_{0}$ has positive scalar curvature, there is a family of diffeomorphisms $\left\{\psi_{t}: M \rightarrow M\right\}$ depending smoothly on $t$ such that $\psi_{t}^{*} g_{t}=g_{0}$.

PROOF. First notice that it suffices to prove the theorem under the assumption that all the $g_{t}$ have positive scalar curvature. For, if not, let $R(t)$ be the (constant) scalar curvature of $g_{t}$, and let $I$ be the largest open interval containing 0 and on which $R(t)$ is positive; $R(t)$ is a continuous function of $t$. If the theorem is true under the additional assumption of positive scalar curvature, $R(t)$ is constant on $I$, and hence positive on some bigger interval if $I \neq \mathbf{R}$. By the maximality of $I$, we must then have $I=\mathbf{R}$.

Let us therefore assume that the $g_{t}$ have positive scalar curvature for $i \in \mathbf{R}$, and let $\pi: \hat{Z} \rightarrow \mathbf{R}$ be the family whose fibers are the twistor spaces $Z_{t}$ of the quaternionic-Kähler manifolds $\left(M, g_{t}\right)$. This is a family of complex contact manifolds satisfying $H^{1}\left(Z_{t}, \mathscr{O}\left(L_{t}\right)\right)=0$ for all $t \in \mathbf{R}$, so that, by the proposition, there is a diffeomorphism $\hat{\psi}: Z_{0} \times \mathbf{R} \rightarrow \hat{Z}$ which sends $Z_{0} \times\{t\}$ biholomorphically onto $Z_{t}$ in a contact-structure preserving fashion. Let $\hat{\psi}_{t}: Z_{0} \rightarrow Z_{t}$ via $\hat{\psi}_{t}(z)=\hat{\psi}(z, t)$.

Now $Z_{t}$ possesses a real structure, meaning an antiholomorphic map $\sigma_{t}: Z_{t} \rightarrow Z_{t}$ without fixed points such that $\sigma_{t}^{2}=1$ and the fibers of $P_{t}: Z_{t} \rightarrow M$ are invariant under $\sigma_{t}$. Let us pull these real structures back to $Z=Z_{0}$ to obtain real structures $\rho_{t}:=\hat{\psi}_{t}^{-1} \sigma_{t} \hat{\psi}_{t}$ on $Z$.

Now $\rho_{t}$ sends the contact distribution to itself, and thus defines an antilinear map of $H^{0}(Z, \mathscr{O}(L))$ to itself. $H^{0}(Z, \mathscr{O}(L))=\mathfrak{g}$ is the Lie algebra of the complex Lie group $G$ of complex contact transformations of $Z$; $\rho_{t}$ is a real form for $\mathfrak{g}$ leaving fixed those infinitesimal complex contact transformations arising from Killing fields on $\left(M, g_{t}\right)$. Since the isometry group of $\left(M, g_{t}\right)$ is compact, $G$ is reductive and $[\mathbf{H}]$ any two such real forms are conjugate via elements of $G$. Thus for all $t$ we can find $\gamma_{t} \in G$ depending continuously on $t$ such that $\rho_{t}=\gamma_{t}^{-1} \rho_{0} \gamma_{t}$ and such that $\gamma_{0}=1$.

Let $\Phi_{t}=\hat{\psi}_{t} \gamma_{t}^{-1}$, so that $\sigma_{0}=\Phi_{t}^{-1} \sigma_{t} \Phi_{t}$. The fibers of $Z_{t}$ are precisely the $\sigma_{t}$-invariant elements of a complete analytic family of compact curves, so that $\Phi_{t}$ sends the fibers of $p_{0}: Z_{0} \rightarrow M$ to the fibers of $p_{t}: Z_{t} \rightarrow M$. Consequently, there are diffeomorphisms $\psi_{t}: M \rightarrow M$ making the diagram

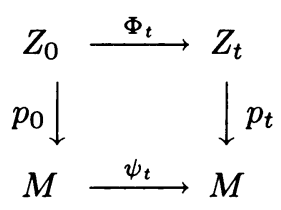

commute. Since $\Phi_{t}$ is a complex contact transformation, and since the contact structure of the twistor space determines the metric up to an overall factor, $\psi_{t}^{*} g_{t}=$ $c_{t} g_{0}$ for some $c_{t}>0$. But since $g_{t}$ and $g_{0}$ have the same volume, $c_{t}=1$ and $\psi_{t}$ is an isometry between $\left(M, g_{0}\right)$ and $\left(M, g_{t}\right)$. Q.E.D. 
REMARKS. (1) The deformation theory of complex contact manifolds given in the proposition may be extended to the case when $H^{1}(X, \mathscr{O}(L)) \neq 0$ but $H^{2}(X, \mathscr{O}(L))=0$; in this case, $H^{1}(X, \mathscr{O}(L))$ becomes the tangent space to a universal family of complex contact deformations of $X$.

(2) Equipped with 20/20 hindsight, one should be able to translate the above proof into terms which avoid using the twistor space. The vanishing of $H^{1}(Z, \mathscr{O}(L))$ corresponds [S2] via the Penrose transform to the triviality of solutions of a linear differential equation on $M$ which apparently are to be interpreted as "linearized quaternionic metrics modulo diffeomorphisms". The Kodaira vanishing argument should correspond to a Bochner vanishing argument on $M$.

ACKNOWLEDGMENT. It is a pleasure to thank Yat-Sun Poon for many useful discussions, and Simon Salamon for his comments on the manuscript.

\section{BIBLIOGRAPHY}

[A] V. I. Arnold, Mathematical methods of classical mechanics, Springer-Verlag, Berlin, 1978.

[H] S. Helgason, Differential geometry, Lie groups and symmetric spaces, Academic Press, New York, 1978.

[K] K. Kodaira, Complex manifolds and deformations of complex structure, Springer-Verlag, Berlin, 1986.

[S] S. Salamon, Quaternionic Kähler manifolds, Invent. Math. 67 (1982), 143-171.

[S2] _ Differential geometry of quaternionic manifolds, Ann. Sci. École Norm. Sup. 19 (1986), 31-55.

Department of Mathematics, State University of NeW York, Stony Brook, NEW YORK 11794 